\title{
Editorial
}

\section{Elan vital, élan létal: one life but multiple deaths}

\author{
G Kroemer $^{\star, 1,2,3}$, AM Tolkovsky ${ }^{4}$ and Z Zakeri ${ }^{5}$
}

\section{Cell Death and Differentiation (2008) 15, 1089-1090; doi:10.1038/cdd.2008.62}

When Richard Lockshin ${ }^{1,2}$ coined the expression 'programmed cell death' (PCD), more than 40 years ago, ${ }^{3}$ he modestly and accurately described the unique morphological and biochemical features of a developmental cell-death process that affected the intersegmental muscles of silkmoths. En passage, he developed the important notion that PCD would be controlled by a combination of cell-extrinsic and cell-intrinsic factors, yet would be executed through a plethora of genetically controlled catabolic reactions, from inside of the self-destroying cell. In retrospect, it is hard to believe that Richard did not foresee that his discovery - a mixture of dedicated and inventive bench work and visionary extrapolation - would be one of the major breakthroughs in cell biology of the 20th century. More interestingly, the process he observed was not apoptosis but one of the emerging new mechanisms of PCD, in a story that is now just unfolding.

The concept of PCD, as initially formulated by Richard and then extended and applied by a panoply of follow-up studies, is most intriguing because it introduces the notion of constructive death into the science of life, biology. Perhaps this is the reason why there has been a strong tendency in the field, in particular between 1980 and 2000, to envisage two simplistic approaches to the problem. First, there has been a tendency to believe that there would be just one single important PCD mechanism in animals, namely apoptosis. Second, based on the discovery of the Caenorhabditis elegans death (CED) genes, many non-specialists have thought that there would be a sort of specialized device, the 'apoptotic machinery', whose building blocks would be exclusively dedicated to the execution of PCD, yet would not have any relevant function in normal life, as if élan vital and élan létal were necessarily opposed and separate entities. Both these (dogmatic) tendencies toward simplification have been broken, and the field is now open again, as open as during the early days when Lockshin translated his microscopic observations into data and concepts. The present special issue of Cell Death Differentiation, which celebrates Richard Lockshin's 70th birthday, perfectly illustrates this regained spirit of freedom.

Although apoptosis undoubtedly represents an important modality of PCD in many animal species, there are other forms of PCD that can be distinguished by simple morpho- logical observation. For example, the linker cell of $C$. elegans succumbs to a PCD that morphologically resembles necrosis and that does not require any among the quintessential apoptotic (CED) genes. ${ }^{4}$ Autophagic processes may also play a major role in the development of the mammalian neural tube, although there may be an important cross talk between apoptotic and autophagic pathways. ${ }^{5}$ Human cancer cells do not only succumb to classical apoptosis when their DNA is damaged by chemotherapy or radiotherapy but also can die through mitotic catastrophe, a process in which cell death occurs during or after a prolonged mitotic arrest, often in cells that have undergone micro- or multinucleation. This mitotic catastrophe can lead to secondary apoptosis and necrosis, and it can be debated whether it constitutes a separate entity of cell death. ${ }^{6}$ Irrespective of semantic issues, it appears that there is not just one single cell-death program and that cells can die through multiple distinct subroutines.

It has become increasingly clear that none of the individual proteins that participate in the major self-destructive events linked to apoptosis, mitochondrial outer-membrane permeabilization (MOMP) and caspase activation, is exclusively dedicated to self-destructive reactions. Rather, all of them also exert some function in normal, death-unrelated processes. Night killers have day jobs. Thus, two archetypical 'cell death genes', egl-1 and ced-4, turned out to play a major role in the adaptation of $C$. elegans cells to starvation and DNA damage, respectively. ${ }^{7}$ Mammalian caspases have also many death-unrelated functions, as illustrated for caspase-8 that participates in the activation of $\mathrm{T}$ and $\mathrm{B}$ lymphocytes, as well as in macrophage differentiation, among other processes. $^{8}$ Caspase-12 turns out to be essential for the induction of cytoprotective autophagy in the context of stress affecting the endoplasmic reticulum. ${ }^{9}$ Bcl-2-like proteins, which regulate the cell death-associated mitochondrial membrane permeabilization as well as mitochondrial fission, have been discovered to play a cardinal role in synaptic plasticity. ${ }^{10}$ The mutation of Itch, an E3 ubiquitin ligase that plays an important role in determining the stability of p73, induces inflammatory lesions as well as lymphoid hyperplasia, illustrating how one single cell-death regulator can affect multiple cellular functions. ${ }^{11}$ Finally, phosphatidylserine exposure, one of the cardinal features of apoptotic cell death, may play an important role in conveying anti-inflammatory

\footnotetext{
${ }^{1}$ INSERM, U848, 39 rue Camille-Desmoulins, Villejuif F-94805, France; ${ }^{2}$ Department of Vivant, Institut Gustave Roussy, Villejuif, France; ${ }^{3}$ Université Paris-Sud, Paris-11, Villejuif, France; ${ }^{4}$ Department of Biochemistry, University of Cambridge, Cambridge, UK and ${ }^{5}$ Department of Biology, Queens College and Graduate Centre of the City University of New York, Flushing, NY, USA

*Corresponding author: G Kroemer, INSERM U848, Institut Gustave Roussy, Pavillon de Recherche 1, 39 rue Camille-Desmoulins, Villejuif F-94805, France.

Tel: + 331421160 46; Fax: + 331421160 47;

E-mail: kroemer@igr.fr
} 
signals to phagocytes. ${ }^{12}$ Altogether, the overall tendency in the field is to reconcile élan vital and élan létal, admitting that molecules that have been initially characterized in the context of apoptosis have other 'normal' functions.

Richard Lockshin used Lepidoptera for his pioneering studies, thereby introducing insects into the field of PCD research. The study of intersegmental cell death has been continued and refined, ${ }^{13}$ yielding ever-increasingly sophisticated mechanistic insights into mammalian death pathways. Moreover, PCD has been investigated in great genetic detail in Drosophila melanogaster, a species in which the contribution of MOMP to cell death is debated, yet still controversial. ${ }^{14}$ However, phylogenetic studies performed in multiple animal species suggest that MOMP-related cytochrome $c$ release, as well as cytochrome $c$-mediated caspase activation, are phylogenetically ancient phenomena that may have been 'invented' by mother nature well before the speciation of insects occurred. ${ }^{15}$ This points to the intriguing possibility that the MOMP-mediated control of caspase activation may have been suppressed during the evolution of some animal species (such as Drosophila and C. elegans), perhaps with the aim of avoiding stress-mediated, unwarranted cell-death events in species that, at the adult stage, are essentially composed by post-mitotic cells and hence cannot afford cell loss.

Altogether, the field of cell-death research is unraveling unexpected hidden treasures. In view of the tremendous clinical importance of cell-death control, ${ }^{16}$ it is just a question of time when the initial observations formulated by studying silkworm intersegmental muscles will crystallize therapeutic developments for the treatment of major diseases. Richard Lockshin's adventure, which started as an innocent, tentative pursuit to understand some particular facet of insect physiology, illustrates how basic concepts in biology may have far-reaching consequences for the conception of (and perhaps for the solution to) human disease-relevant processes.

Acknowledgements. GK is supported by a special grant from LNC, as well as grants by ANR, ANRS, Cancéropôle lle-de-France, FRM, European Commission and INCa; ZZ is supported by a grant from the National Institutes of Health. AMT is supported by the Wellcome Trust.

1. Lockshin R, Williams CM. J Insect Physiol 1964; 10: 643-649.

2. Lockshin R. Cell Death Differ 2008; 15: 1091-1095 (this issue).

3. Zakeri Z. Cell Death Differ 2008; 15: 1087-1088 (this issue).

4. Blum ES, Shaham S. Cell Death Differ 2008; 15: 1124-1131 (this issue)

5. Cecconi F, Piacentini M, Fimia GM. Cell Death Differ 2008; 15: 1170-1177 (this issue).

6. Vakifahmetoglu H, Olsson M, Zhivotovsky B. Cell Death Differ 2008; 15: 1153-1162 (this issue).

7. Galluzzi L, Joza N, Tasdemir E, Maiuri MC, Hengartner M, Abrams JM et al. Cell Death Differ 2008; 15: 1113-1123 (this issue)

8. Wallach D, Kang T-B, Kovalenko A. Cell Death Differ (in press).

9. Py BF, Lipinski MM, Sohn EJ, Saad NB, Zhu H, Yuan J. Cell Death Differ (in press).

10. Berman SB, Pineda F, Hardwick JM. Cell Death Differ 2008; 15: 1147-1152 (this issue).

11. Melino G, Galagher E, Peschiaroli A, Rossi M, Scialpi F, Malatesta M et al. Cell Death Differ 2008; 15: 1103-1112 (this issue).

12. Birge RB, Ucker DS. Cell Death Differ 2008; 15: 1096-1102 (this issue).

13. Schwartz LM. Cell Death Differ, 15: 1163-1169 (this issue).

14. Steller H. Cell Death Differ 2008; 15: 1132-1138 (this issue).

15. Oberst A, Bender C, Green DR. Cell Death Differ 2008; 15: 1139-1146 (this issue)

16. Butts BD, Houde C, Mehmet H. Cell Death Differ 2008; 15: 1178-1186 (this issue). 